\title{
NEW MINIMUM-COMPONENT OTA-BASED SINUSOIDAL OSCILLATORS
}

\author{
MUHAMMAD TAHER ABUELMA'ATTI AND MUHAMMAD \\ HAROON KHAN
}

King Fahd University of Petroleum and Minerals, Box 203, Dhahran 31261, Saudi Arabia

(Received March 21, 1995; in final form May 5, 1995)

Using a general oscillator structure, new oscillator circuits are systematically derived. These oscillator circuits use two operational transconductance amplifiers (OTAs) and one externally connected passive element, exploiting the inherent zeros of the OTAs to advantage. The feasibility of obtaining oscillation using only two OTAs without externally connected passive elements is investigated. Experimental results are included.

\section{INTRODUCTION}

At present, operational-transconductance-amplifier (OTA)-based oscillators are receiving considerable attention. This is attributed mainly to their electronic tunability over a wide-range, the use of a relatively small number of active and passive components, the feasibility of obtaining relatively high-frequencies of oscillation, and finally their suitability for integration in CMOS and bipolar technology. Although the literature [1]-[6] contains a large number of attractive OTA-based oscillator circuits, the proposed circuits are analyzed assuming ideal OTAs, that is, the OTA is acting as an ideal voltage-controlled current-source (VCCS). OTAs are, however, non-ideal in many respects. Of particular interest here is the output conductance, the output and input capacitances and the frequency dependence of the transconductance [7] and [8]. The equivalent circuit model of a non-ideal OTA operating in the linear region is shown in Fig. 1. Although the influence of the OTA non-idealities of Fig. 1 on the performance of OTA-based oscillators has been studied [2]-[4], no attempt has been reported to exploit these OTA non-idealities to advantage. Of particular interest here is the utilization of the frequency dependent transconductance of practical OTAs to advantage. This may result in designs with minimum externally-connected passive components.

It is the major intention of this paper to show that new minimum-component OTA-based sinusoidal oscillators can be obtained by exploiting, to advantage, the frequency-dependence of the OTA transconductance. In this regard, two new OTA-based oscillator circuits will be presented. Each circuit uses two OTAs and one externally-connected passive element (a resistor or a capacitor). The feasibility of obtaining oscillators using OTAs only without any externally-connected passive elements will be investigated. 

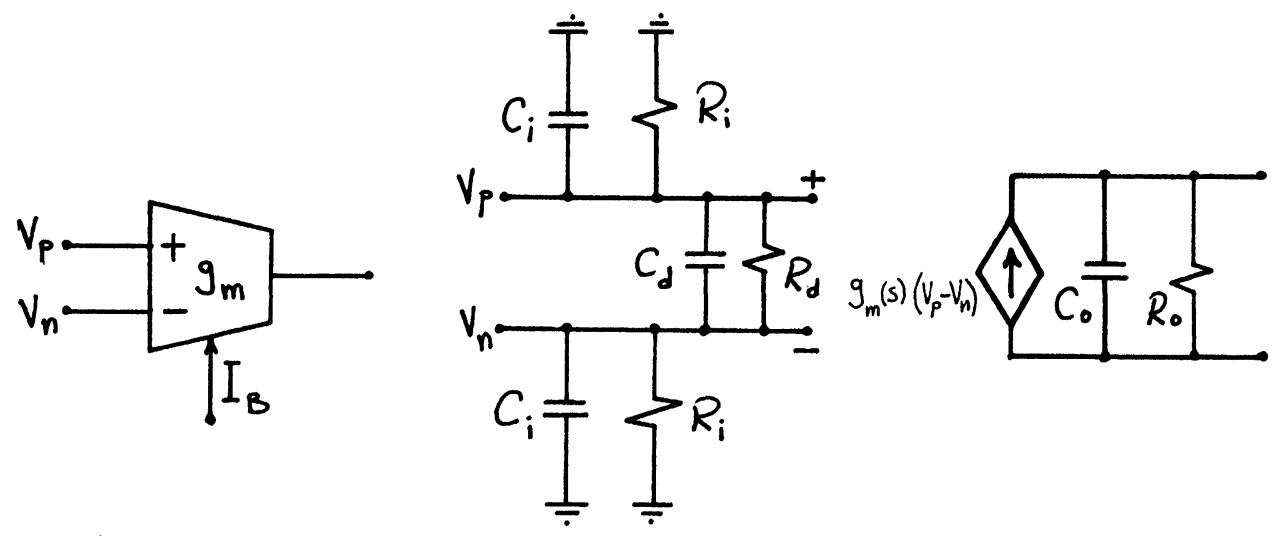

FIGURE 1 Circuit symbol (a) and circuit model (b) of a nonideal OTA operating in the linear region.

\section{PROPOSED CIRCUITS}

Consider the oscillator structure shown in Fig. 2. Assuming that the OTAs are non-ideal and can be represented by the circuit model of Fig. 1, then the equivalent circuit of the oscillator structure of Fig. 2 will be as shown in Fig. 3, where $Z_{T 1}$ is the parallel combination of $R_{o 1}, R_{i 2}, R_{d 2}$ and $\left(C_{o 1}+C_{i 2}+C_{d 2}\right)$ and $Z_{T 2}$ is the parallel combination of $R_{o 2}, R_{i 1}, R_{d 1}$, and $\left(C_{o 2}+C_{i 1}+C_{d 1}\right)$. The model takes into consideration the frequency dependence of the transconductance, $g_{m}(s)$, which can be expressed as [8]

$g_{m i}(s)=g_{m o i} \exp \left(-s T_{i}\right)$

where $T_{i}$ is the time delay through and $g_{m o i}$ is the dc transconductance of the ith OTA. For frequencies much lower than $\omega_{z i}=1 / T_{i},(1)$ can be approximated by

$g_{m i}(s)=g_{m o i}\left(1-s / \omega_{z i}\right)$

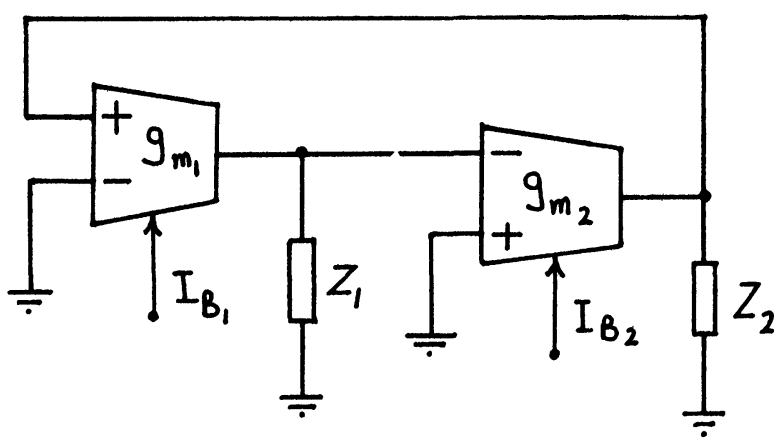

FIGURE 2 Proposed oscillator structure. 
where $\omega_{z i}$ is the frequency of the single-zero model of the ith OTA. Although (2) is not particularly exact, it allows us to obtain simple expressions for the frequency and the condition of oscillation of the proposed oscillator structure.

Routine analysis yields the characteristic equation of the equivalent circuit of Fig. 3 as

$g_{m 1} g_{m 2} \frac{Z_{1} Z_{2} Z_{T 1} Z_{T 2}}{\left(Z_{1}+Z_{T 1}\right)\left(Z_{2}+Z_{T 2}\right)}+1=0$

Substituting

$Z_{T 1}=\frac{R_{T 1}}{1+s C_{T 1} R_{T 1}}, Z_{T 2}=\frac{R_{T 2}}{1+s C_{T 2} R_{T 2}}$

where $C_{T 1}=C_{o 1}+C_{i 2}+C_{d 2}, R_{T 1}=R_{o 1} / / R_{i 2} / / R_{d 2}, C_{T 2}=C_{o 2}+C_{i l}+C_{d 1}, R_{T 2}=$ $R_{o 2} / / R_{i 1} / / R_{d 1}$, and approximating the transconductance of the ith OTA by (2), equation (3) reduces to

$\frac{g_{m o 1} g_{m o 2}\left(\omega_{z 2}-s\right)\left(\omega_{z 1}-s\right) R_{T 1} R_{T 2} Z_{1} Z_{2}}{\left(R_{T 2}+Z_{2}+s C_{2} R_{T 2} Z_{2}\right)\left(R_{T 1}+Z_{1}+s C_{T 1} R_{T 1} Z_{1}\right) \omega_{z 1} \omega_{z 2}}+1=0$

By performing all possible permutations to explore the possibility of obtaining sinusoidal oscillations using one grounded passive component only, the two oscillator circuits shown in Fig. 4 resulted. The frequency of oscillation and the condition of oscillation for each circuit are given in Table I.

From Table I, it is obvious that no oscillation can be obtained if the OTAs are assumed ideal with $\omega_{z 1}=\omega_{z 2}=\infty$. Moreover, if

$g_{m o 1} g_{m o 2} R_{T 1} R_{T 2} \gg 1+R_{T 1} / R_{1}$

and

$C_{T 1} C_{T 2} \gg g_{m o 1} g_{m o 2} / \omega_{z 1} \omega_{z 2}$

then the frequency of oscillation of the circuits of Fig. 4 will be given by

$\omega_{o}^{2}=g_{m o 1} g_{m o 2} / C_{T 1} C_{e q}$

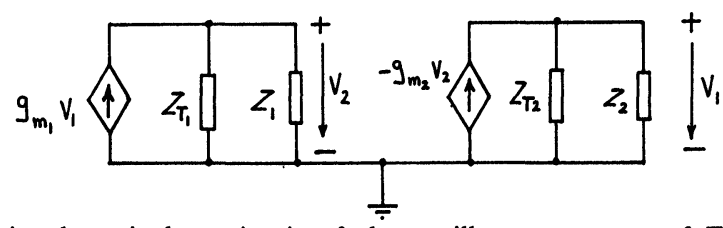

FIGURE 3 Small signal equivalent circuit of the oscillator structure of Fig. 2. $Z_{\mathrm{T} 1} \equiv$ Parallel Combination of: $R_{\mathrm{o} 1}, \mathbf{R}_{\mathrm{i} 2}, \mathbf{R}_{\mathrm{d} 2},\left(\mathrm{C}_{\mathrm{o} 1}+\mathrm{C}_{\mathrm{i} 2}+\mathrm{C}_{\mathrm{d} 2}\right) \mathrm{Z}_{\mathrm{T} 2} \equiv$ Parallel Combination of: $\mathbf{R}_{\mathrm{o} 2}, \mathbf{R}_{\mathrm{i} 1}, \mathbf{R}_{\mathrm{d} 1},\left(\mathrm{C}_{\mathrm{o} 2}+\right.$ $\mathrm{C}_{\mathrm{i} 1}+\mathrm{C}_{\mathrm{d} 1}$. 


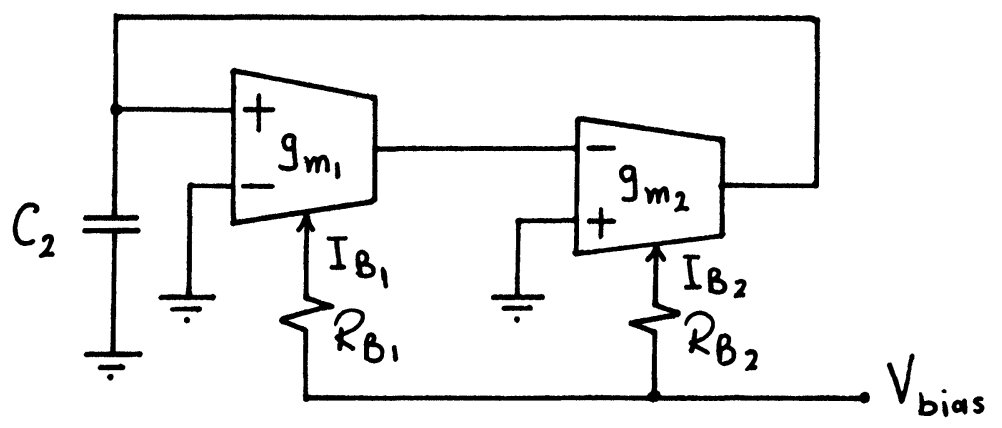

(a)

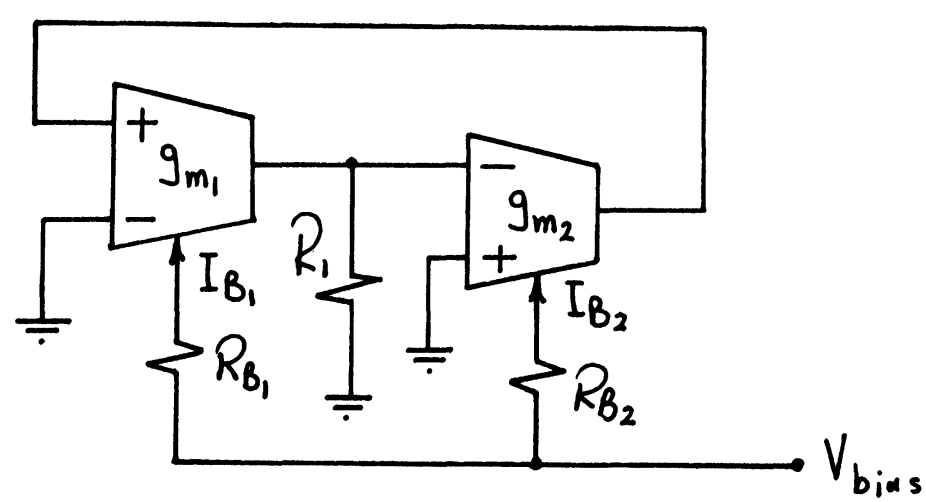

(b)

FIGURE 4 Oscillator circuits derived from the proposed structure of Fig. 2.

where $C_{e q}=C_{T 2}+C_{2}$ for the circuit of Fig. 4(a) and $C_{e q}=C_{T 2}$ for the circuit of Fig. 4(b).

Inspection of (6) and (7) shows that the expected frequency of oscillation, $\omega_{o}^{2}$, is subject to the constraint $\omega_{o}^{2}<<\omega_{z 1} \omega_{z 2}$. This is compatible with the approximate model used for the transconductance of the OTA. Moreover, from equation (7) it

TABLE I

Frequency and Condition of Oscillation

\begin{tabular}{|c|c|c|}
\hline Circuit & Frequency of Oscillation, $\omega_{0}{ }^{2}$ & Condition of Oscillation \\
\hline \multirow{2}{*}{ Fig. 4(a) } & $1+g_{m o 1} g_{m o 2} R_{T 1} R_{T 2}$ & $g_{m o l} g_{m o 2} R_{T 1} R_{T 2}$ \\
\hline & $R_{T l} R_{T 2}\left(\frac{g_{m o l} g_{m o 2}}{\omega_{z l} \omega_{z 2}}+C_{T l}\left(C_{2}+C_{T 2}\right)\right)$ & $\omega_{z 1}+\omega_{z 2}-\left(C_{2}+C_{T 2}\right) R_{T 2}+C_{T 1} R_{T 1}$ \\
\hline \multirow{2}{*}{ Fig. 4(b) } & $R_{T I}+R_{I}+g_{m o l} g_{m o 2} R_{T l} R_{T 2} R_{I}$ & $g_{m o l} g_{m o 2} R_{l} R_{T l} R_{T 2}$ \\
\hline & $\overline{R_{T l} R_{T 2} R_{l}\left(\frac{g_{m o l} g_{m o 2}}{\omega_{z l} \omega_{z 2}}+C_{T l} C_{T 2}\right)}$ & $\overline{C_{T 2} R_{T 2}\left(R_{T 1}+R_{l}\right)+C_{T 1} R_{T 1} R_{l}}$ \\
\hline
\end{tabular}


is obvious that if the transconductances of the OTAs are varied simultaneously through a single external current [9] and [10], then $g_{m o 1}=g_{m o 2}=g_{m o}$ and (7) reduces to

$\omega_{o}=g_{m o} /\left(C_{T I} C_{e q}\right)^{1 / 2}$

For OTAs with bipolar differential input stage, $g_{m o}$ can be expressed by $g_{m o}=$ $I_{B} / 2 V_{T}$ where $I_{B}$ is the amplifier bias current of each OTA and $V_{T}$ is the thermal voltage, and equation (8) reduces to

$\omega_{o}=\frac{I_{B}}{2 V_{T}\left(C_{T 1} C_{e q}\right)^{1 / 2}}$

From equation (9), one can set that as long as the capacitances $C_{T 1}$ and $C_{e q}$ are independent of the OTA bias current, $I_{B}$, then the frequency of oscillation of the circuits of Fig. 4 can be linearly controlled by a single external current.

From Table I, one can see that if $C_{2}=0$, the circuit of Fig., 4(a) can yield oscillation using only two OTAs. Similar results can be obtained from the circuit of Fig. 4(b) if $R_{1}=\infty$. In both cases, the frequency of oscillation will be given by

$\omega_{o}^{2}=\frac{1+g_{m o 1} g_{m o 2} R_{T 1} R_{T 2}}{R_{T 1} R_{T 2}\left(g_{m o 1} g_{m o 2} / \omega_{z 1} \omega_{z 2}+C_{T 1} C_{T 2}\right)}$

If the conditions of equations (5) and (6) are satisfied, then the frequency of oscillation will be given by equation (7).

\section{EXPERIMENTAL RESULTS}

The circuits of Fig. 4 were experimentally tested using the LMA3080 IC OTAs. Some experimental results are shown in Fig. 5 for $R_{1}=10 \mathrm{~K}$ and $C_{2}=1.47 n F$. From Fig. 5(a), one can see that the frequency of oscillation is not changing linearly with the amplifier bias currents, while from Fig. 5(b), the frequency of oscillation is changing linearly with the amplifier bias current. This is attributed to the dependence of the parasitic elements $C_{T 1}, C_{T 2}, R_{T 1}$, and $R_{T 2}$ on the amplifier bias current. For the LM3080 OTA, the parasitic capacitances $C_{T 1}$ and $C_{T 2}$ are nearly constant for $I_{B i} \leq 30 \mu A$ [11]. For higher values of $I_{B i}$, the parasitic capacitances are no longer constant. Moreover, the parasitic resistances $R_{T 1}$ and $R_{T 2}$ decrease rapidly as the amplifier bias current increases [11]. Thus, inequality (5) may not be satisfied and (7) may not be applicable for relatively large values of the amplifier bias current. Therefore, one may expect that for values of amplifier bias currents $I_{B i}>30 \mu A$, the variation of the frequency of oscillation with the amplifier bias currents will be slightly non-linear. Figure 6 shows an output waveform obtained from the circuit of Fig. 4(a) using two OTAs only without any externally connected passive elements. With $I_{B 1}=I_{B 2}=0.2 \mu A, C_{i 1}=C_{i 2}=C_{o 1}=C_{o 2}=C_{d 1}=C_{d 2} \cong$ $5 p F$, using (9) the expected frequency of oscillation is $42 \mathrm{kHz}$. However, the 
measured frequency is $8.5 \mathrm{kHz}$. This difference is attributed to the oscilloscope and cable capacitances, which may amount to about $120 \mathrm{pF}$ plus additional stray capacitances resulting from the wiring in a discrete-element breadboard realization. Better agreement between theoretical and experimental results is expected in an integrated circuit implementation, where the effect of stray capacitances can be minimized.

\section{DISCUSSION AND CONCLUSION}

In this paper, two new OTA-based oscillators have been presented. The circuits exploit to advantage the frequency dependence of the OTAs. This results in sinusoidal oscillator circuits using only one externally connected passive element (either a resistor or a capacitor). The proposed circuits can easily yield linear current(voltage)-controlled oscillators. The feasibility of obtaining oscillations using OTAs only was also investigated and nearly sinusoidal oscillations were successfully obtained.

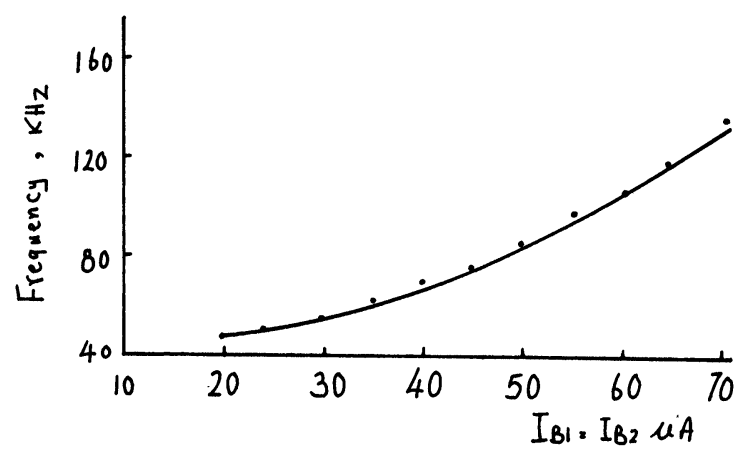

(a)

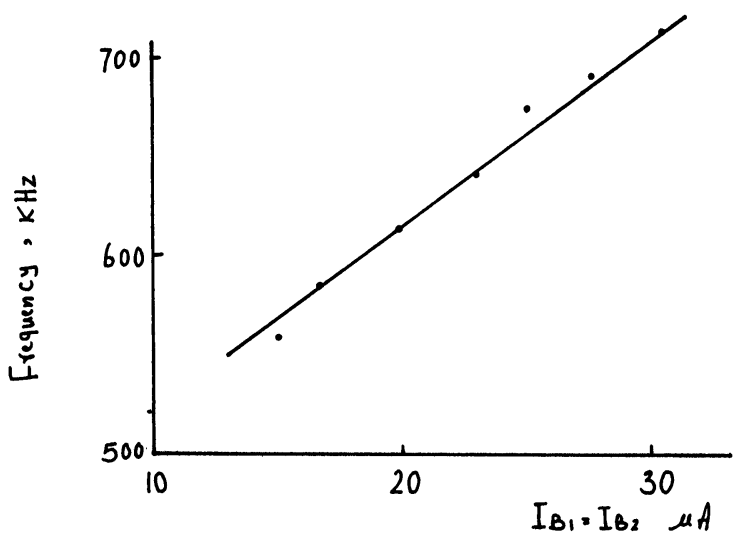

(b)

FIGURE 5 Experimental results obtained from: (a) Fig. 4(a) with $C_{2}=1.47 \mathrm{nF}$ (b) Fig. 4(b) with $R_{1}$ $=10 \mathrm{~K}$. 


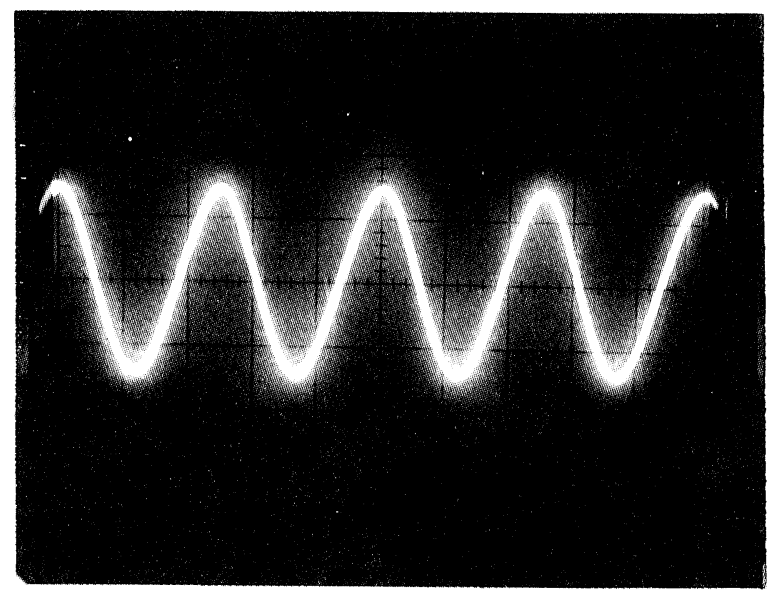

$$
f_{0}=8.5 \mathrm{kHz}, V_{p-p} \simeq 60 \mathrm{mV}
$$

FIGURE 6 Typical output waveform obtained from Fig. 4 (a) with: $C_{2}=0, I_{B 1}=I_{B 2}=0.2 \mu A$.

The frequency and the condition of oscillation of the proposed circuits are interdependent and both are functions of the parasitic elements of the OTA. These parasitic elements are functions of the amplifier bias-current, which, in turn, is a function of temperature. This makes the amplifier bias-current stabilization against temperature drifts very important in practical applications, particularly under varying environmental conditions. Possible solutions for this problem include temperature compensation of the OTAs $[12,13]$ and phase-lock $\omega_{o}$-stabilization techniques [14-17]. Although the later is more suited for relatively complicated systems, it has the advantage of stabilizing system performance not only against temperature variations, but also against variations in power-supply voltage and aging [18].

The major practical problem of the proposed oscillator structures is the interdependence between the frequency and the condition of oscillation. Thus, any attempt to change the frequency of oscillation by changing the amplifier biascurrent may result in changing the condition of oscillation and consequenctly large variations in distortion may be expected unless some form of non-linear amplitude control, other than the inherent non-linearity of the OTA, is used.

Despite the above mentioned practical problems, the simplicity of the proposed circuits deserve attention. They are very attractive in applications where the waveshape and frequency stability are not very stringent.

\section{REFERENCES}

1. D.R. Bhaskar, M.P. Tripathi and R. Senani, A class of three-OTA-two-capacitor oscillators with non-interacting controls, International Journal of Electronics, Vol. 74, 1993, pp. 459-463

2. B. Linares-Barranco, A. Rodrigues-Vazquez, E. Sanchez-Sinencio and J.L. Heurtas, Generation, 
design and tuning of OTA-C high frequency sinusoidal oscillators, IEE Proceedings, Part G, Vol. 139,1992 , pp. 557-568

3. B. Linares-Barranco, A. Rodrigues-Vazquez, E. Sanchez-Sinencio and J.L. Huertas, CMOS OTA-C high-frequency sinusoidal oscillators, IEEE Journal of Solid-State Circuits, Vol. 26, 1991, pp. $160-165$

4. A. Rodrigues-Vazquez, B. Linares-Barranco, J.L. Heurtas and E. Sanchez-Sinencio, On the design of voltage-controlled sinusoidal oscillators using OTAs, IEEE Transactions on Circuits and Systems, Vol. 37, 1990, pp. 198-211

5. D.R. Bhaskar, M.P. Tripathi and R. Senani, Systmatic derivation of all possible canonic OTA-C sinusoidal oscillators, Journal of the Franklin Institute, Vol. 330, 1993, pp. 885-603

6. I.A. Khan, M.T. Ahmed and N. Minhaj, A simple realisation scheme for OTA-C universal biquadratic filter, International Journal of Electronics, Vol. 72, 1992, pp. 419-429

7. A. Rodrigues-Vazquez, B. Linares-Barranco, J.L. Heurtas, E. Sanchez-Sinencio and J.J. Hoyle, Generation and design sinusoidal oscillator using OTAs, Proceedings IEEE International Symposium on Circuits and Systems, 1988, pp. 2863-2866

8. H. Nevarez-Lozano, J.A. Hill and E. Sanchez-Sinencio, Frequency limitations of continuous-time OTA-C filters, Proceedings IEEE International Symposium on Circuits and Systems, 1988, pp. 2169-2172

9. R. Senani, New elwectronically tunable OTA-C sinusoidal oscillator, Electronics Letters, Vol. 25, 1989, pp. 286-287

10. R.L. Geiger and E. Sanchez-Sinencio, Active filter design using operational transconductance amplifiers: A tutorial, IEEE Circuits and Devices Magazine, Vol. 1, 1985, pp. 20-32

11. Linear Data Book, National Semiconductor Corporation, 1982, pp. 2-485/2-488

12. C.A. Karybakas, C. Kosmatopoulos and Th. Laopoulos, Improved temperature compensation of OTAs, Electronics Letters, Vol. 28, 1992, pp. 763-764

13. H.S. Malvar and M. Leuttgen, Temperature compensation of OTA-based filters and amplifiers, Electronics Letters, Vol. 23, 1987, pp. 890-891

14. J.R. Brand, R. Schaumann and E.M. Skei, Temperature stabilized active-R filters, Proceedings of the 20th Midwest Symposium on Circuits and Systems, 1977, pp. 295-300

15. K.R. Rao, V. Sethuraman and P.K. Neelakantan, A novel "Follow the master" filter, Proceedings IEEE, Vol. 65, 1977, pp. 1725-1726

16. J.R. Brand and R. Schaumann, Comments on "A nover'Follow the master' filter", Proceedings IEEE, Vol. 66, 1978, pp. 590-592

17. K.S. Tan and P.R. Gray, High-order monolithic analog filters using bipolar/JFET technology, Digest of the IEEE International Solid-State Circuits Conference, 1978, pp. 80-81

18. J.R. Brand and R. Schaumann, Active R filters: review of theory and practice, IEE Journal of Electronic Circuits and Systems, Vol. 2, 1978, pp. 89-101 

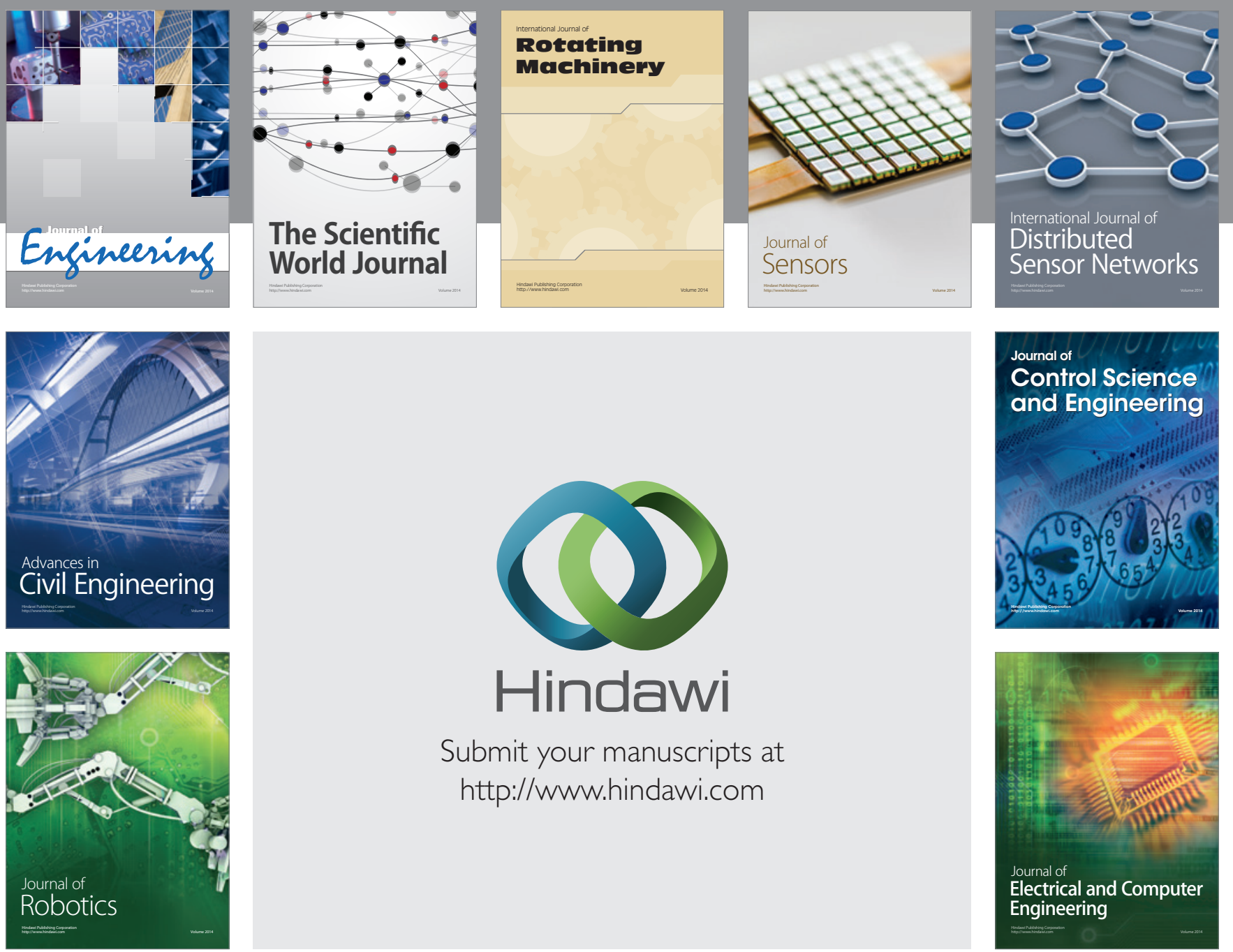

Submit your manuscripts at

http://www.hindawi.com
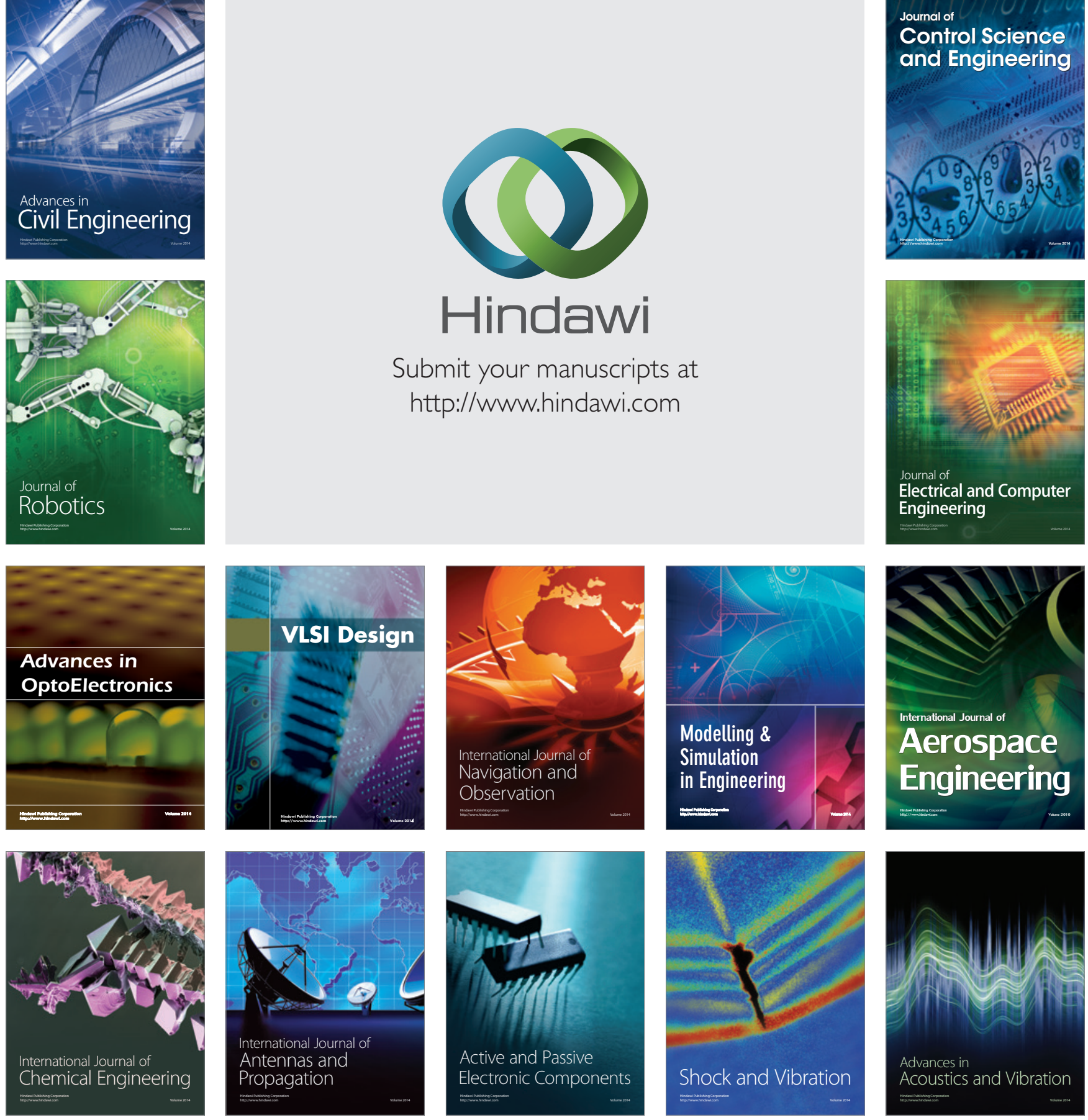